\section{MS37-P5 A racemic solution of an organometallic complex crystallizing as a disordered crystal structure of opposite enantiomers (pseudoracemate) in a chiral space group}

Roy Herrmann ${ }^{1}$, Beatrice Braun ${ }^{1}$, Thomas Braun ${ }^{1}$

1. Humboldt-Universität zu Berlin, Brook-Tayol-Str. 2, 12489 Berlin (Germany)

email: roy.herrmann@chemie.hu-berlin.de

The crystallization of a racemic solution results generally in three types of crystalline racemates: racemic compounds, racemic conglomerates, or pseudoracemates (see Figure). The most encountered type of crystalline racemates are racemic crystals (heterochiral) in which both $S$ and $R$ enantiomers are present in an equal amount in the unit cell in a well-ordered manner. Additionally, as a result of spontaneous resolution in $S$ and $R$ handed crystals, conglomerates (homochiral) can be obtained. A rare outcome of the crystallization of a racemic solution is the formation of pseudoracemic crystals (heterochiral), in which both $S$ and $R$ enantiomers are present in the crystal structure in a disordered manner. ${ }^{[1]}$ A Pseudoracemate is a solid solution of opposite enantiomers which can crystallize in any space group. Their existence was first formulated by Kipping and Pope. ${ }^{[2]}$

All three types of crystalline racemates differ in their melting point diagrams and properties. That is why their accurate characterization is crucial for the pharma industry in the context of chiral drug design. Due to the fact that in general, only one of the enantiomers has biological activity, the other being inactive, inhibiting the desired effect or even being toxic. We were fortunate to come across such structure with a 1:1 stoichiometry of opposite enantiomers. Particularly interesting and extraordinarily rare is the fact that our pseudoracemate crystallizes in a Sohnke space group, therefore making a heterochiral crystal crystallize in a chiral space group. The compound under discussion is an organometallic complex crystallizing as a THF solvate in the monoclinic chiral space group $P 2$. The asymmetric unit contains one independent complex molecule on general position, whereas no other particularly interesting intra- or intermolecular interactions are present.

[1] H. D. Flack, Helv. Chim. Acta 2003, 86, 905-921.

[2] F. S. Kipping, W. J. Pope, J. Chem. Soc. Trans. 1897, 71, 989-1001.

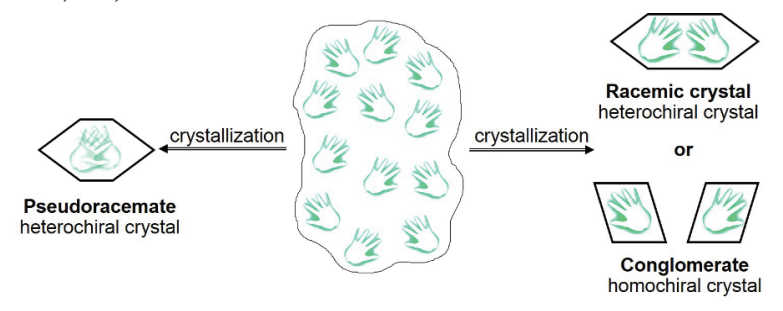

Figure 1. Crystallization types of a racemic solution.

Keywords: chirality, racemate, pseudoracemate, organometallic compounds

\section{MS37-P6 X and N multi-temperature study on rotational disorder in crystalline N-Acetyl-L4-hydroxyproline \\ Jens Luebben ${ }^{1,2}$, Birger Dittrich ${ }^{2}$, Alison Edwards ${ }^{3}$}

1. Georg-August-University Goettingen, Tammanstrasse 4, 37077 Goettingen, Germany

2. University Hamburg, Martin-Luther-King-Platz 6, 20146 Hamburg, Germany

3. Australian Nuclear Science and Technology Organisation, New Illawarra Road, Lucas Heights, Australia

email: jens.luebben@stud.uni-goettingen.de

Molecular crystals of N-Acetyl-L4-hydroxyproline and $\mathrm{N}$-Acetyl-L4-hydroxyproline monohydrate show rotational disorder of acetyl hydrogen atoms at different temperatures. [1, 2] The anhydrate is disordered at temperatures above $200 \mathrm{~K}$ while the monohydrate form shows disorder at temperature above $100 \mathrm{~K}$. A multi temperature study using X-Ray and neutron diffraction was carried out to understand better the conditions that cause this effect and the effect of disorder in general.

The identical chemical bonding in both compounds and the similar crystal packing make $\mathrm{N}$-Acetyl-L4-hydroxyproline an ideal candidate for this study.

Several neutron data sets between $9 \mathrm{~K}$ and $250 \mathrm{~K}$ of both forms were collected [3] and studied along with their $\mathrm{X}$-Ray counterparts and molecular dynamics simulations to gain in-depth understanding of the dynamic processes in the crystalline state.

[1] M. Hospital, C. Courseille, F. Leroy, B. P. Roques, Biopolymers 18, 1141-1148 (1979).

[2] B. Dittrich, J. E. Warren, F. P. A. Fabbiani, W. Morgenroth, B. Corry, Phys. Chem. Chem. Phys., 11, 2601-2609 (2009).

[3] J. Lubben, C. Volkmann, S. Grabowsky, A. Edwards, W. Morgenroth, F. P. A. Fabbiani, G. M. Sheldrick and B. Dittrich, Acta Cryst. A70 , 309-316 (2014).

Keywords: Neutron Diffraction, Low Temperature, Disorder 